\title{
Interleukin-17 aggravates right ventricular remodeling via activating STAT3 under both normoxia and hypoxia
}

\author{
Jing Huang ${ }^{1}$, Wei Zhang ${ }^{2}$, Cai-lian Zhang ${ }^{3}$ and Lei Wang ${ }^{4^{*}}$
}

\begin{abstract}
Objective: Proinflammatory cytokine interleukin 17 (IL-17) is involved in ventricular remodeling, mainly of the left ventricle. This study was designed to explore the role of IL-17 played in the pathogenesis of right ventricular hypertrophy (RVH), aiming to provide a novel treatment target or diagnostic biomarker options for improving the care of RVH patients.

Methods: C57BL/6 mice were maintained in 10\% $\mathrm{O}_{2}$ chamber or room air for four weeks. Right ventricular hypertrophy index (RVHI), RV/body weight ratio, pulmonary arteriolar remodeling determined by percent media thickness $(\% \mathrm{MT})$, and the cardiomyocyte diameter of RV were evaluated. Mice were treated with exogenous recombinant mouse IL-17 (rmlL-17, $1 \mu \mathrm{g}$ per dose twice a week) for four weeks. H9c2 cardiomyocytes were cultured and treated with IL-17 $(10 \mathrm{ng} / \mathrm{mL})$ and STAT3 inhibitor $(10 \mathrm{ng} / \mathrm{mL})$ either under normoxia $\left(21 \% \mathrm{O}_{2}, 5 \% \mathrm{CO}_{2}, 74 \% \mathrm{~N}_{2}\right)$ or under hypoxia ( $3 \% \mathrm{O}_{2}, 5 \% \mathrm{CO}_{2}, 92 \% \mathrm{~N}_{2}$ ). Cardiomyocyte viability was assessed by Cell counting kit 8 (CCK-8) assay. The mRNA level was detected by RT-PCR, where as the protein expression was measured by Western blot, immunohistochemistry, and immunofluorescent analyses.
\end{abstract}

Results: In vivo experiments showed that IL-17 did not affect the pulmonary artery under normoxia, after treatment with rmIL-17, \%MT was not changed, while RVHI and the RV/body weight ratio were increased, indicating that IL-17 directly induced right ventricular hypertrophy. In a time-course study, the mice were exposed to hypoxia for 0, 1, 2, 3, 4 weeks, respectively. We found that the expression of IL-17 was gradually upregulated in RV tissue in a time-dependent manner after one week of hypoxia exposure, especially at the third and fourth week. Cardiomyocyte hypertrophy and apoptosis were observed after the exposure of the mice to hypoxia for four weeks, rmIL-17 further aggravated the hypoxia-induced cardiomyocyte hypertrophy and apoptosis. The expression of p-STAT3 in the IL-17-deficient mice was lower than in the wild-type mice. In vitro, IL-17 inhibited cardiomyocyte viability and induced cardiomyocyte apoptosis via STAT3 under both normoxic and hypoxic conditions.

Conclusions: These findings support a role for IL-17 as a mediator in the pathogenesis RVH, which might be considered as a potential novel anti-inflammation therapeutic strategy or diagnostic biomarker for RVH.

Keywords: Right ventricular hypertrophy, cardiomyocyte apoptosis, IL-17, STAT3

*Correspondence: wl860806wb@163.com

${ }^{4}$ Department of Pulmonary and Critical Care Medicine, Second Affiliated Hospital of Xi'an Jiaotong University, No. 157 Xiwu Road, Xin Cheng District, Xi'an 710004, People's Republic of China

Full list of author information is available at the end of the article Jing Huang and Wei Zhang are co-first author

\section{Introduction}

Right ventricular hypertrophy (RVH) results from adaptation of right ventricle (RV) to an increased afterload and biomechanical stress, ultimately leading to right heart failure (RHF) and death, mainly observed 
in pulmonary hypertension [1]. The main pathophysiological characteristics of RVH are cardiomyocyte hypertrophy, elevated capillary density and synthesis of extracellular matrix proteins [2], which is a remodeling process. In contrast to the left ventricle (LV), RV rapidly switches from adaptive to maladaptive RVH and finally to end-stage heart failure, leading eventually to death. However, no specific biomarkers for RV failure and no treatment have been indentified so far that specifically address RV dysfunction. Therefore, an urgent need exists to clarify the potential pathogenesis of RVH.

$\mathrm{RVH}$ is a complex and heterogeneous disease, and the RV remodeling of this disease is not well elucidated. Many factors are reportedly involved in RVH, including ion channels, neurohormonal activation, metabolism dysfunction, myocardial perfusion, genetic factors, inflammation and extracellular matrix changes [2]. Among them, inflammation and immune activation critically involved in pulmonary vascular and RV remodeling $[3,4]$. Furthermore, inflammation, immune mediator and immune cell activities, such as neutrophils, macrophage and lymphocytes infiltration, contribute to the remodeling and the development of RVH [5-8].

Evidence suggested that $\mathrm{CD} 4^{+}$helper $\mathrm{T}$ (Th) cells participate in inflammatory heart disease, and the deficiency in $\mathrm{CD} 4^{+}$Th cells induced ventricular remodeling $[9,10]$. The number of $\mathrm{CD} 4^{+}$Th cells subset Th17 cells infiltrated in the LV was increased significantly in the heart failure rabbit model [9]. The important pro-inflammation cytokine IL-17 mainly secreted by Th17 cells, has been reported to participate in the cardiac ventricular remodeling in some heart diseases, such as ischemic heart failure [9], myocardial infarction [11], and dilated cardiomyopathy [12]. While previous studies have predominantly focused on the role of IL-17 in the left ventricle remodeling, very few studies have investigated its involvement in RVH. Thus, the role of IL-17 in RVH remains to be elucidated (Additional file 1).

Therefore, in this study, we examined the role of IL-17 in the pathogenesis of RV remodeling and the possible signal pathways involved to provide a novel treatment target or diagnostic biomarker options for improving the care of RVH patients.

\section{Materials and methods}

\section{Animal models}

The experimental animals were approved by the Institutional Animal Care and Use Committee of Xi'an jiaotong University. Animals were housed in accordance with the regulations for the Management of Laboratory Animals published by the Ministry of Science and Technology of the People's Republic of China. Eight-week old male C57BL/6N mice weighing 20-25 g were bought from Vital River Laboratory Animal Technology Company (Beijing, China), IL17 ${ }^{-1-}$ male mice (C57BL/6N background) aged 8 weeks and their matched wide type (WT) littermates were a kindly gift from Professor Dai of China-Japan Friendship Hospital (Beijing, China). All the mice were housed in a $12 \mathrm{~h}$ light/12 $\mathrm{h}$ dark cycle, and food and water were available ad libitum. Mice were allowed to acclimate for 3 days and were then exposed to normobaric hypoxia $\left(10 \% \mathrm{O}_{2}\right)$ in an airtight plexiglass chamber for 4 weeks. Control mice were exposed to room air under the same conditions.

\section{Treatments}

For exogenous IL-17 administration experiments, recombinant murine IL-17 (rmIL-17, peprotech, NJ, USA) dissolved in PBS was administered intraperitoneally at a dose of $1 \mu \mathrm{g} /$ mouse per dose twice a week for consecutive 4 weeks, the control groups were given equal volume of PBS [13]. For experiments of different time points for hypoxia, the mice were sacrificed at $0,1,2,3$, or 4 weeks, respectively. The flowcharts of in vivo experiments can be seen in Additional file 1.

\section{Cells culture}

H9c2 cardiomyocytes were purchased from Procell Life Science \& Technology (CL-0089, Wuhan, China) and subsequently kept in DMEM with 10\% (v/v) FBS, $100 \mathrm{U} /$ $\mathrm{mL}$ penicillin and $100 \mathrm{~g} / \mathrm{mL}$ streptomycin in a humidified normoxia condition $\left(21 \% \mathrm{O}_{2}, 5 \% \mathrm{CO}_{2}, 74 \% \mathrm{~N}_{2}\right)$ at $37^{\circ} \mathrm{C}$. Cells were passaged (passages 4-6) after reaching 80-90\% confluence, detached with $0.05 \%$ trypsin, $0.04 \%$ EDTA (Sigma-Aldrich, MO, USA) in phosphate-buffered saline (PBS). For hypoxia injury, the cells were maintained at $37{ }^{\circ} \mathrm{C}$ in a humidified hypoxia condition $\left(3 \% \mathrm{O}_{2}\right.$, $\left.5 \% \mathrm{CO}_{2}, 92 \% \mathrm{~N}_{2}\right)$ for $24 \mathrm{~h}$, and treated with IL-17 (10 ng/ $\mathrm{ml})$ or STAT3 inhibitor $(10 \mathrm{ng} / \mathrm{ml})$.

\section{CCK-8 assay}

To evaluate cell viability, H9c2 cells were seeded in 96-well plates and tested via CCK- 8 assay (Dojindo, Kumamoto, Japan). After addition 10 ul of CCK- 8 solution in each well, cells were then cultured. After $4 \mathrm{~h}$, the absorbance of $450 \mathrm{~nm}$ was monitored via microplate reader (Thermo, MULTISKAN MK3).

\section{Measurement of right ventricular hypertrophy}

The method was described in previous papers $[14,15]$. Mice were anesthetized with $2 \%$ pentobarbital $(50 \mathrm{mg} /$ $\mathrm{kg}$, i.p.). The mice were then sacrificed, and the hearts were collected. To evaluate the extent of right ventricle (RV) hypertrophy, RV tissues were separated and the weights of the RV and the left ventricle (LV) plus interventricular septum (S) were measured respectively. 
The right ventricular hypertrophy index (RVHI) was defined as: RVHI $(\%)=[\mathrm{RV} /(\mathrm{LV}+\mathrm{S})] \times 100$. The ratio of RV weight to body weight (BW) was also calculated.

Cardiomyocyte cross-sectional diameter was determined in the RV as measures of cardiac ventricle tissue remodeling. $4 \mu \mathrm{m}$ sections of the RV were stained with H\&E. Microscopic images were analyzed in a blinded manner using Nikon microscope digital camera system and its image analysis program (Nikon, Tokyo, Japan).

\section{Lung artery morphometric analysis}

The method was described in previous papers [13, 16]. The paraffin-embedded lungs were serially sectioned at a thickness of 4 um for morphometric analysis. Images of pulmonary arterioles were captured with an Olympus microscope digital camera system (Olympus, Tokyo, Japan), and the arterial circumferences were measured using the Image Pro Plus 5.1 image analysis program (Media Cybernetics, Silver Spring, MD). The pulmonary arterioles with external diameters smaller than $100 \mu \mathrm{m}$ accompanied by either alveolar ducts or alveoli were measured. Pulmonary arteriolar remodeling was estimated by percent media thickness (MT\%), $\mathrm{MT} \%=\left(\right.$ circumference $_{\text {ext }} / \pi$ - circumferenceint $\left._{\text {int }} / \pi\right) /$ (circumference $\left._{\text {ext }} / \pi\right) \times 100$ [17], and circumference ${ }_{\mathrm{ext}}$ and circumference $_{\text {int }}$ mean the circumferences bounded by the external and internal elastic lamina.

\section{RT-PCR}

RT-PCR was performed as previously conducted in our laboratory [16]. Total RNA was extracted from cultured cells using Trizol reagents (Sigma-Aldrich, USA). Reverse transcription was performed using Superscript III Firststrand Synthesis System (Invitrogen, USA) and quantitative, real-time PCR with Power SYBR Green PCR Master Mix (Applied Biosystems, UK). The relative abundance of target mRNA was normalized to that of the GAPDH, by a comparative cycle threshold method $\left(2^{-\Delta \Delta C T}\right)$. The primer sequences were as listed below:

GAPDH: Forward: 5'-ACAGCAACAGGGTGGTGG AC $-3^{\prime}$, and Reverse: $5^{\prime}$-TTTGAGGGTGCAGCGAAC TT-3';

Bax: Forward: 5'-CAGGCGAATTGGCGATGAAC -3', and Reverse: $5^{\prime}$-CCCAGTTGAAGTTGCCGTCT-3';

Bcl2: Forward: 5'-GCCTTCTTTGAGTTCGGTGG -3', and Reverse: 5'-CTGAGCAGCGTCTTCAGAGA - ${ }^{\prime}$;

Caspase-3: Forward: 5'-TGGACTGCGGTATTGAGA CA $-3^{\prime}$, and Reverse: 5'-GCGCAAAGTGACTGGATG AA $-3^{\prime}$.

\section{Immunohistochemical analysis}

IL-17 expression levels were detected in paraffin-embedded mouse right ventricular tissue sections using the rabbit anti-mouse IL-17 (Abcam, UK, 1:50 dilution) antibodies. Protein expression was visualized using HRPconjugated goat anti-rabbit secondary antibody ( $\& \& D$, CA, USA, 1:200 dilution). The positive cells were developed by diaminobenzidine (DAB) reagent, nuclei were counterstained with hematoxylin.

\section{Immunofluorescent analysis}

IL-17, Bax and caspase-3 expression levels were detected in paraffin-embedded RV sections using the anti-IL17 antibody (Abcam, UK, 1:20 dilution), anti-Bax antibody (Proteintech, Wuhan, China, 1:100 dilution) and anticaspase-3 antibody (Bioss, Beijing, China, 1:200 dilution), protein expression was visualized using Alex Fluor 488 Goat Anti-Rabbit secondary antibodies (R\&D, CA, USA, 1:200 dilution, green) or Cy3 Conjugated Goat Anti-Rabbit IgG secondary antibody (BOSTER biological technology, Wuhan, China, 1:100 dilution, red). Nuclei were counterstained with DAPI.

\section{Western blot analysis}

Proteins were separated by SDS-PAGE and transferred to nitrocellulose membranes. Membranes were blocked in $5 \%$ non-fat dry milk for $1 \mathrm{~h}$, followed by incubation in anti-IL-17 (rabbit, Affinity Biosciences, OH, USA, 1:1000 dilution), anti-p STAT3 (rabbit, Affinity Biosciences, $\mathrm{OH}$, USA, 1:1000 dilution), anti-GAPDH (rabbit, Goodhere Biological Technology, China, 1:1000 dilution), anti-Bax (rabbit, proteintech, Wuhan, China, 1:3000 dilution), anti-Bcl2 (rabbit, proteintech, Wuhan, China, 1:600 dilution) and anti-caspase3 (rabbit, Cell Signaling Technology, MA, USA, 1:1000 dilution) antibodies overnight at $4{ }^{\circ} \mathrm{C}$. After the overnight incubation, membranes were washed with TBST buffer and re-incubated with a goat anti-rabbit IgG-HRP secondary antibody. The membranes were washed with TBST buffer and reacted with the electrochemiluminescence (ECL) substrate (Thermo, MA, USA) and exposed to X-ray film. The value of the relative density of each target protein band was normalized to the density of the corresponding GAPDH band.

\section{Statistical analysis}

Data were presented as means \pm SD. Unpaired Student's t-test was used for comparisons between two groups. One-way ANOVA with the Bonferroni's multiple comparisons test was used to evaluate differences between more than two groups. $p<0.05$ was considered statistically significant. 


\section{Results}

Under normoxia, IL-17 induced right ventricular hypertrophy but not pulmonary vascular remodeling

We previously confirmed that IL-17 aggravated hypoxia-induced pulmonary vascular remodeling and the secondary right ventricular hypertrophy (RVHI and RV/body weight ratio) that resulted from increased afterload [13]. Here, we investigated whether IL-17 induced pulmonary vascular remodeling under normoxia and its effect on right ventricular. After treating the mice with recombinant mouse IL-17 (rmIL-17) for four weeks, pulmonary artery morphometrics data were analyzed. Vascular remodeling of $\mathrm{PH}$ was observed mainly in the distal pulmonary arteriole, and thus \% MT of $<100 \mu \mathrm{m}$ in the intra-pulmonary arterioles were calculated. The results (Fig. 1A) showed that rmIL-17 did not affect the \% MT of the pulmonary arterioles under normoxia. We also evaluated the right ventricle of the mice. Interestingly, RVHI and RV/body weight ratio, two of the indexes of the right ventricular hypertrophy, were significantly increased after the mice
A

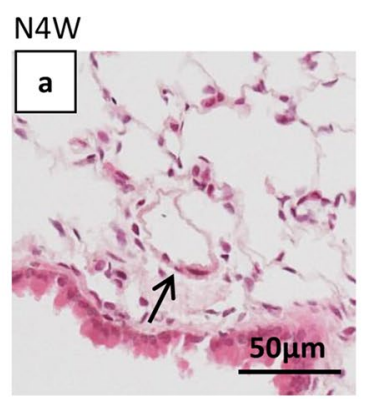

B a

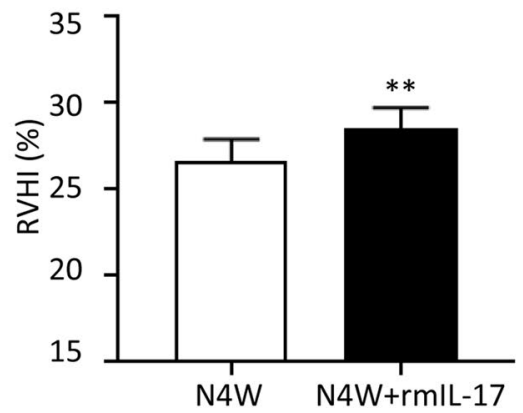

C

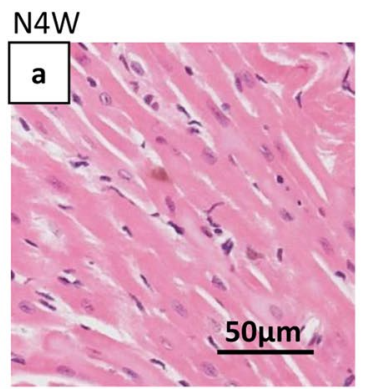

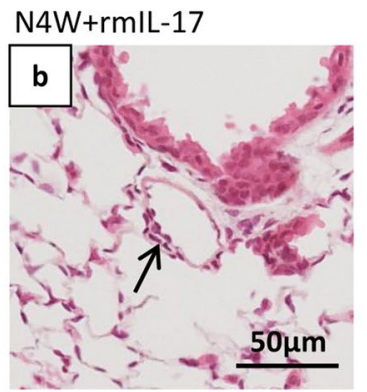

b
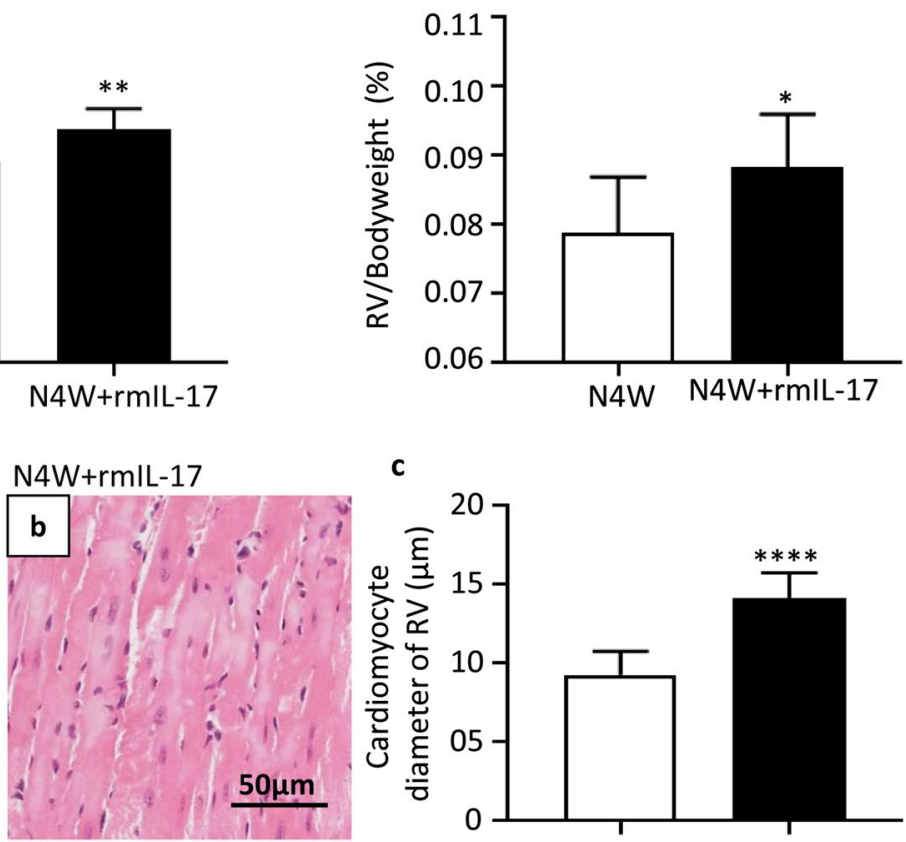
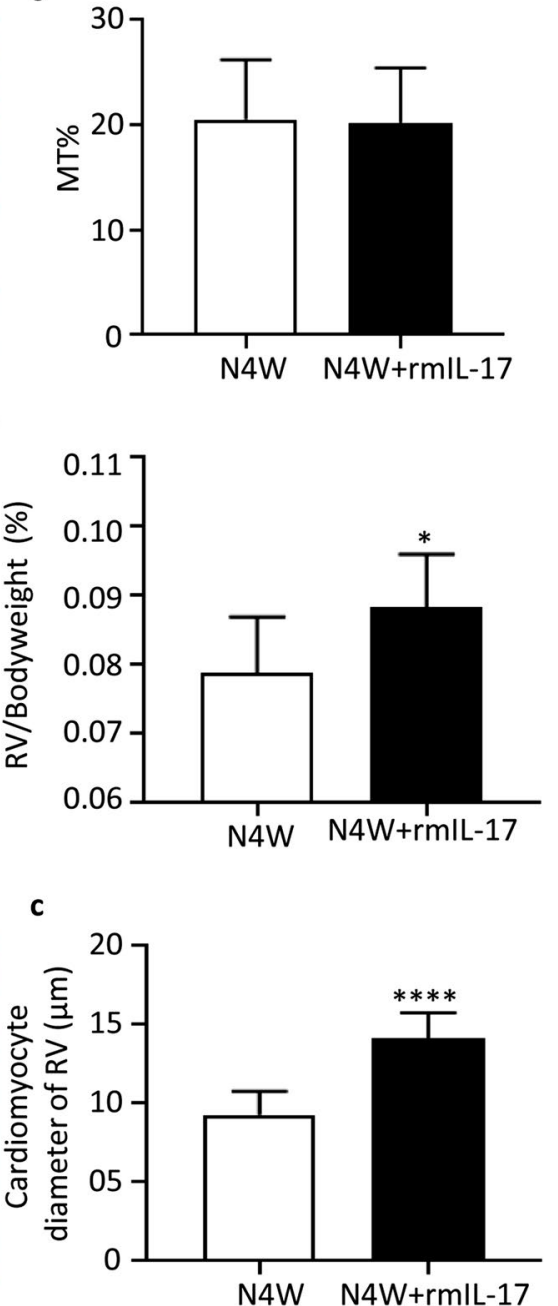

Fig. 1 IL-17 induces right ventricular hypertrophy under normoxia. A Comparison of pulmonary vascular remodeling in mice under conditions of normoxia for 4 weeks (N4W, $n=9$ ) with or without recombinant mouse IL-17 (rmlL-17) (N4W + rmIL-17, $n=9)$. (a, b) H\&E staining of pulmonary arterioles. The arrows show pulmonary arterioles. Scale bars: 50 m. (c) Percentage medial thicknesses (MT\%) of pulmonary arterioles less than $100 \mu \mathrm{m}$. B Right ventricular hypertrophy of mice in the groups of N4W and N4W + rmIL-17. (a) RVHI of mice. RVHI: right ventricular hypertrophy index. ${ }^{* *} p=0.0053$. (b) RV/body weight ratio of mice. ${ }^{*} p=0.0220$. C Cardiomyocyte hypertrophy of the right ventricular tissue sections derived from mice in the groups of N4W and N4W + rmIL-17. (a, b) Representative H\&E staining. (c) Bar chart for cardiomyocytes diameter in the right ventricle of each group. ${ }^{* * *} p<0.0001$ 
under normoxia treated with rmIL-17, as showed in the Fig. 1B. Compared with normoxia mice, rmIL-17 treatment increased the diameter of cardiomyocyte significantly (Fig. 1C).

\section{The expression of IL-17 was upregulated during the chronic hypoxia exposure}

We have already known that RVHI level were gradually increased in a time-dependent manner after hypoxia exposure, and the expression of IL-17 in lung tissue and serum were also gradually increased [13]. To determine the effects of hypoxia exposure on the expression of IL-17 in the right ventricular tissue, RV tissues from mice exposed to hypoxia for $0,1,2,3$, and 4 weeks were collected. Immunohistochemical analysis (Fig. 2A) results revealed that the expression of IL-17 was gradually increased in a time-dependent manner after one week of hypoxia exposure, especially at the third and fourth weeks. The immunofluorescent analysis results (Fig. 2B) further confirmed that the expression of $\mathrm{IL}-17$ was upregulated during the chronic hypoxia exposure.

\section{IL-17 aggravated hypoxia-induced right ventricular hypertrophy}

From the above results, we established that IL-17 could induce right ventricular hypertrophy under normoxia and IL-17 in RV tissue was upregulated during the chronic hypoxia exposure. Our previous data showed that IL-17 aggravated hypoxia-induced right ventricular hypertrophy with elevated RVHI and RV/body weight ratio [13]. Cardiomyocyte hypertrophy and cardiomyocyte apoptosis are two different cellular events in ventricular hypertrophy. Then, we investigated the effect of IL-17 on the cardiomyocyte diameter and apoptosis under hypoxia. Figure 3A illustrates the results of the representative $H \& E$ staining of mice under normoxia, hypoxia treated with IL-17 or not for four weeks (N4W, H4W, H4W + rmIL-17). The cardiomyocyte diameter was measured as an indicator of ventricular hypertrophy (Fig. 3B). The hypoxia-exposed mice had a significantly larger cardiomyocyte diameter than the normoxia mice, which was further promoted by the treatment with rmIL-17. The cardiomyocyte apoptosis was investigated by detecting apoptosis-related gene Bax and caspase-3, as showed in Fig. 3C, the hypoxia-exposed mice had a higher expression of Bax and caspase-3, which was further upregulated by the treatment with rmIL-17. These

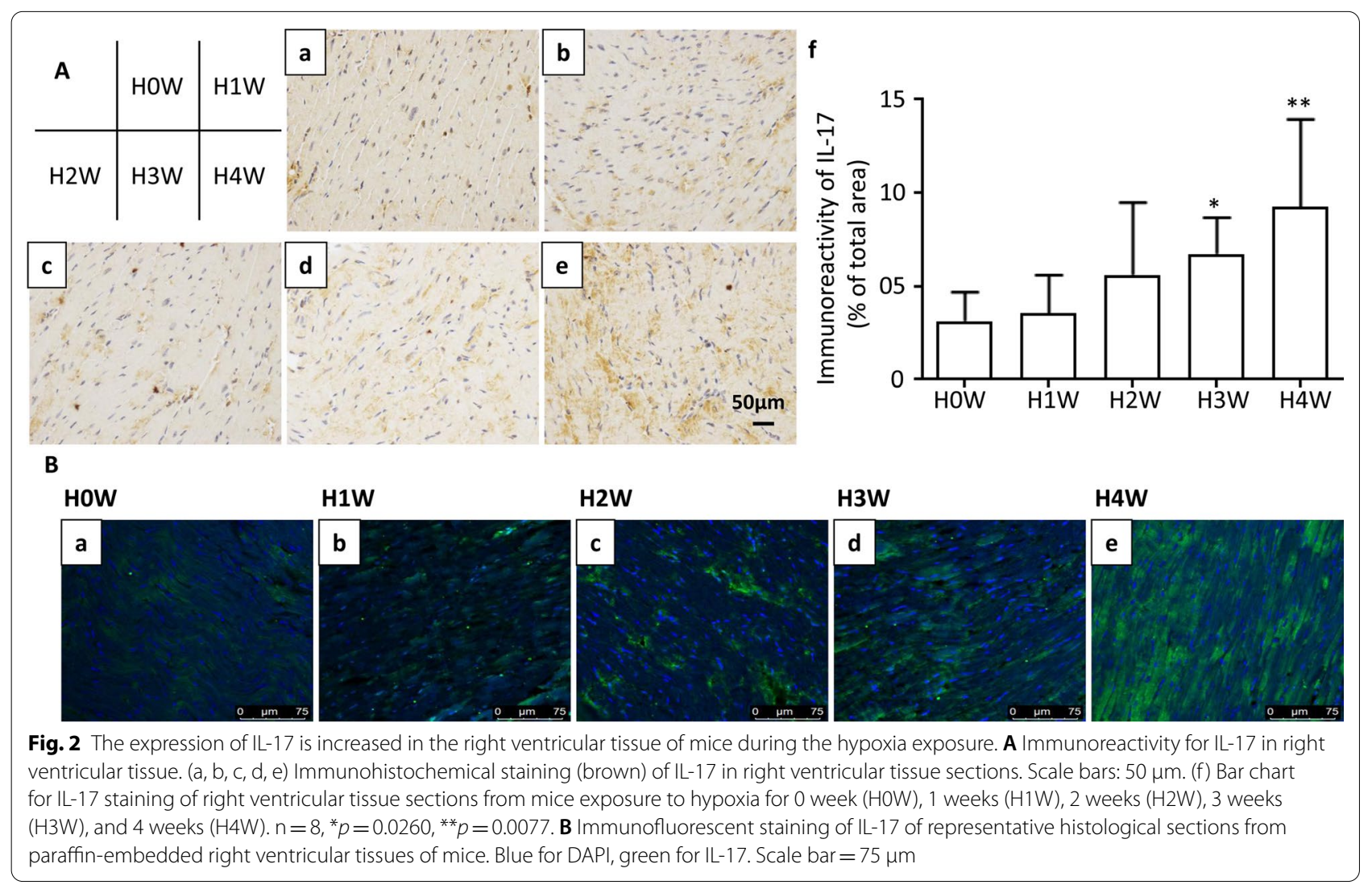


A
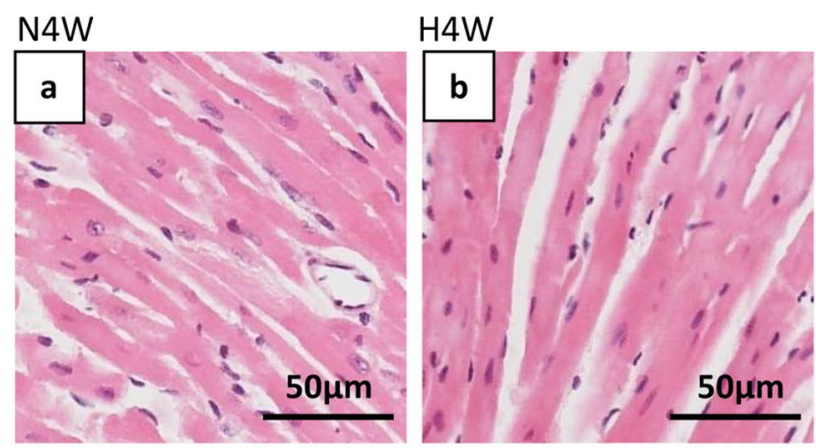

$\mathrm{H} 4 \mathrm{~W}+\mathrm{rmIL}-17$

B

D
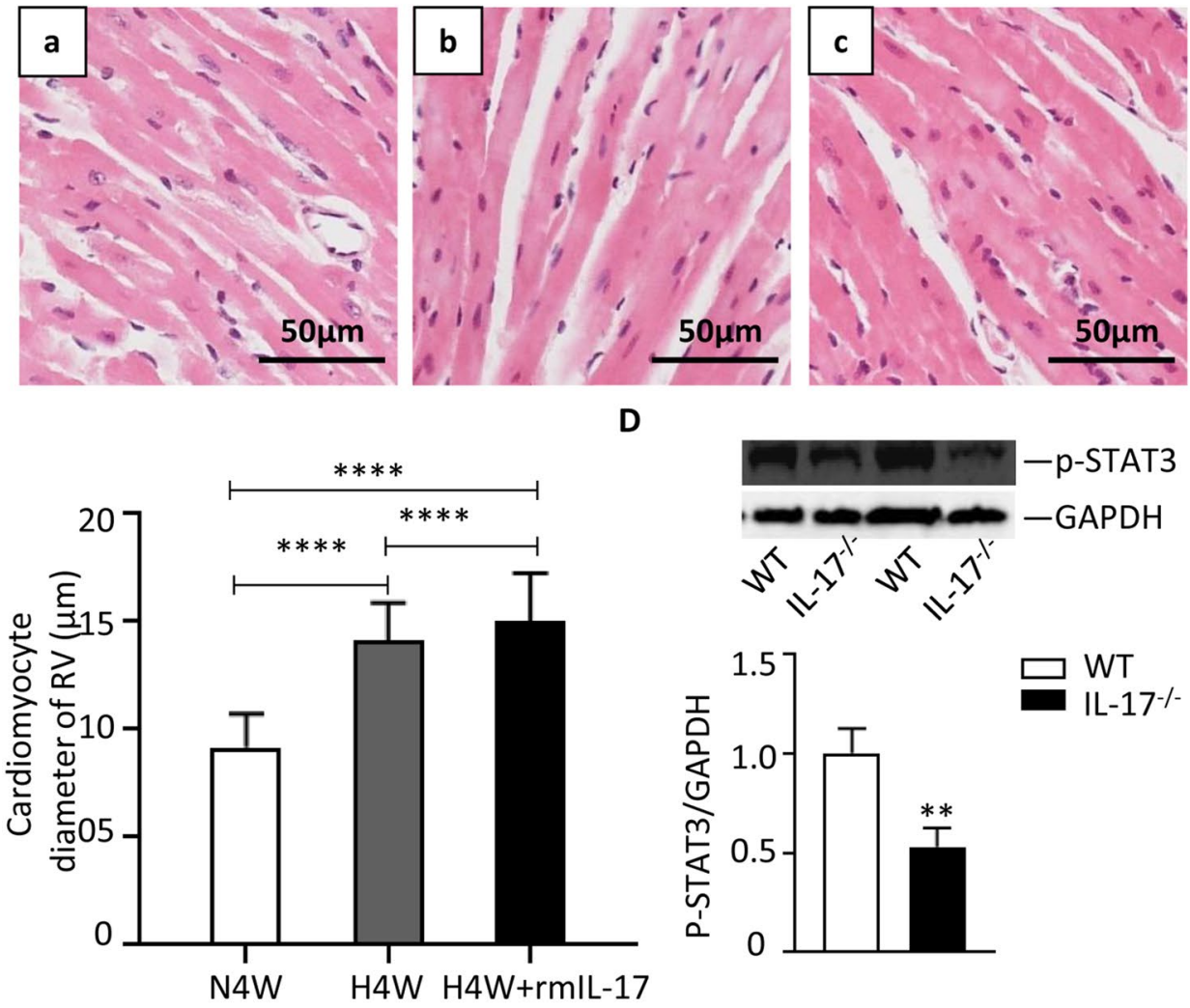

C

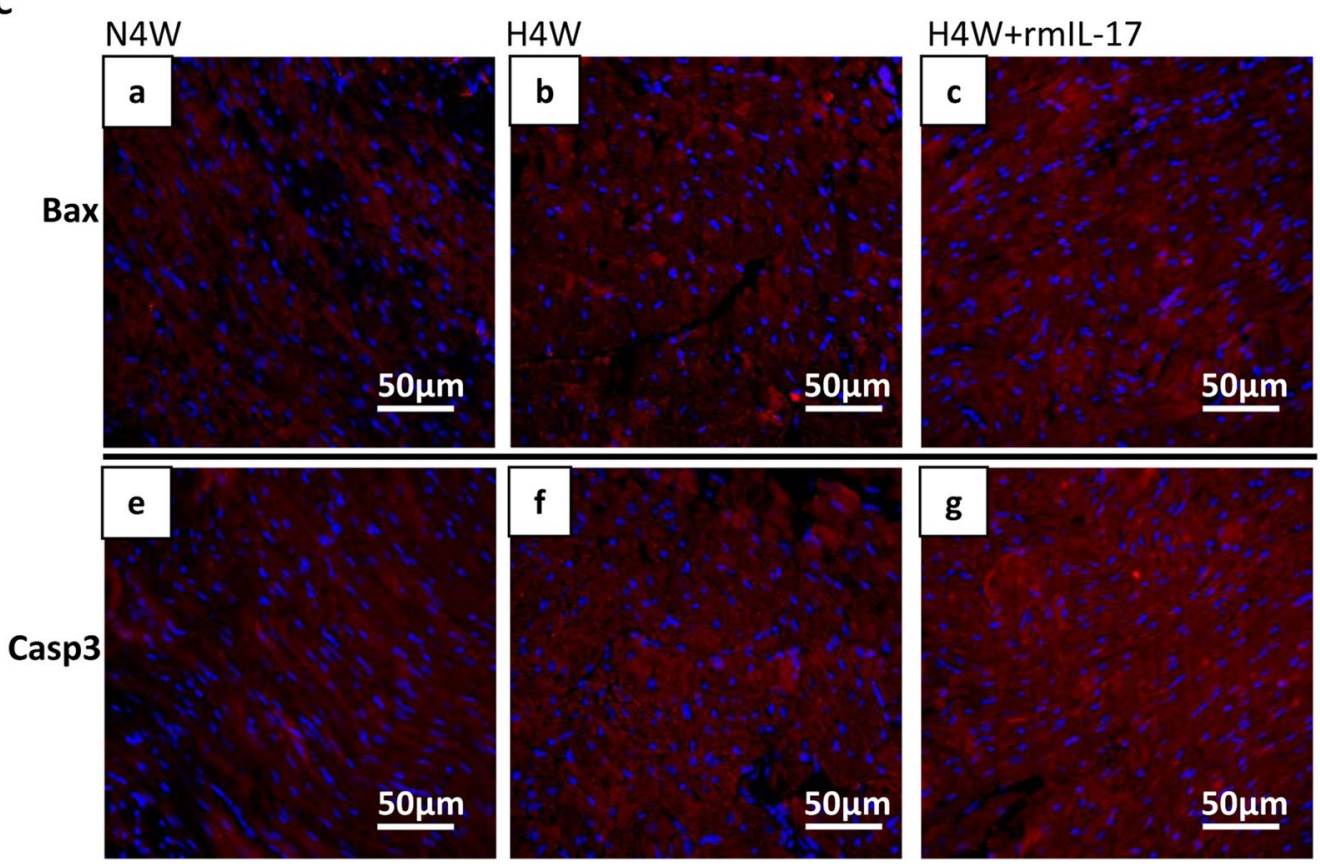

Fig. 3 IL-17 aggravates hypoxia-induced right ventricular hypertrophy through activating STAT3. A Representative H\&E staining of the right ventricular tissue sections derived from mice under normoxia $(N 4 W, n=7)$ or hypoxia $(H 4 W, n=8)$ for 4 weeks with $r m I L-17(H 4 W+r m I L-17, n=6)$ or not. Scale bars: $50 \mu \mathrm{m}$. B Bar chart for cardiomyocytes diameter in the right ventricle of each group. ${ }^{* * *} p<0.0001$. C Immunofluorescent staining of Bax and caspase-3 of representative histological sections from paraffin-embedded mice right ventricular tissues of each group. Blue for DAPI, red for Bax and caspase-3. Scale bar = $50 \mu \mathrm{m}$. D Western blot analysis of p-STAT3 in right ventricular tissue of wide type mice and IL-17 knockout mice $(\mathrm{n}=6)$ and the statistical bar graph. ${ }^{* *} p=0.0063$ 
data show that rmIL-17 significantly aggravated the hypoixa-triggered hypertrophy and apoptosis of individual cardiomyocytes in RV.

\section{The role of STAT3 in the IL-17 induced-right ventricular hypertrophy}

Then, we aimed to better understand the potential mechanism of IL-17 induced-right ventricular injury. We have proved previously that IL-17 deficiency prevented development of hypoxia-induced pulmonary hypertension and right ventricular hypertrophy [13], results in the present study showed that the expression of p-STAT-3 in RV tissues from the IL-17-deficiency mice was lower than that in the wild-type mice (Fig. 3D), indicating that IL-17 may induce right ventricular hypertrophy through STAT-3 activation.

\section{The role of IL-17/STAT-3 in the cardiomyocyte injury}

Cardiomyocyte injury plays an important role during the right ventricular hypertrophy, and hypoxia could cause cardiomyocyte injury [18], which is in agreement with our results. In this study, the expression of IL-17 (Fig. 4A) and p-STAT-3 (Fig. 4B) after hypoxia exposure was upregulated. Notably, the expression of p-STAT-3 was further upregulated by the IL-17 treatment (Fig. 4B). As can be observed in Fig. 4C, cardiomyocyte viability was inhibited by IL-17 under both normoxic and hypoxic conditions, which was reversed by the STAT-3 inhibitor.

The effect of IL-17 on cardiomyocyte apoptosis was also investigated by detecting apoptosis-related genes. $\mathrm{Bax}$ and $\mathrm{Bcl}-2$ belong to the $\mathrm{Bcl}-2$ gene family, Bax is a pro-apoptotic regulator, whereas Bcl-2 is an anti-apoptotic regulator, and caspase- 3 is a critical executioner of apoptosis. As illustrated in Fig. 5, IL-17 upregulated the expression of Bax and caspase- 3 but downregulated the expression of $\mathrm{Bcl}-2$ under normoxic and hypoxic conditions, which was reversed by the STAT- 3 inhibitor. These data indicate that IL-17 inhibited cardiomyocyte viability and induced cardiomyocyte apoptosis via STAT-3 under both normoxic and hypoxic conditions.

\section{Discussion}

In this study, we obtained the following main findings: (1) IL-17 induced RVH even under normoxia; (2) The expression of IL-17 in RV tissues during the chronic hypoxia exposure was upregulated in a time-dependent manner; (3) IL-17 further aggravated hypoxia-induced right ventricular hypertrophy; (4) IL-17 might participate in RVH through STAT3 activation; and (5) IL-17 induced cardiomyocyte injury and further aggravated hypoxiainduced cardiomyocyte injury by activating STAT3. To our knowledge, this is the first report to demonstrate that

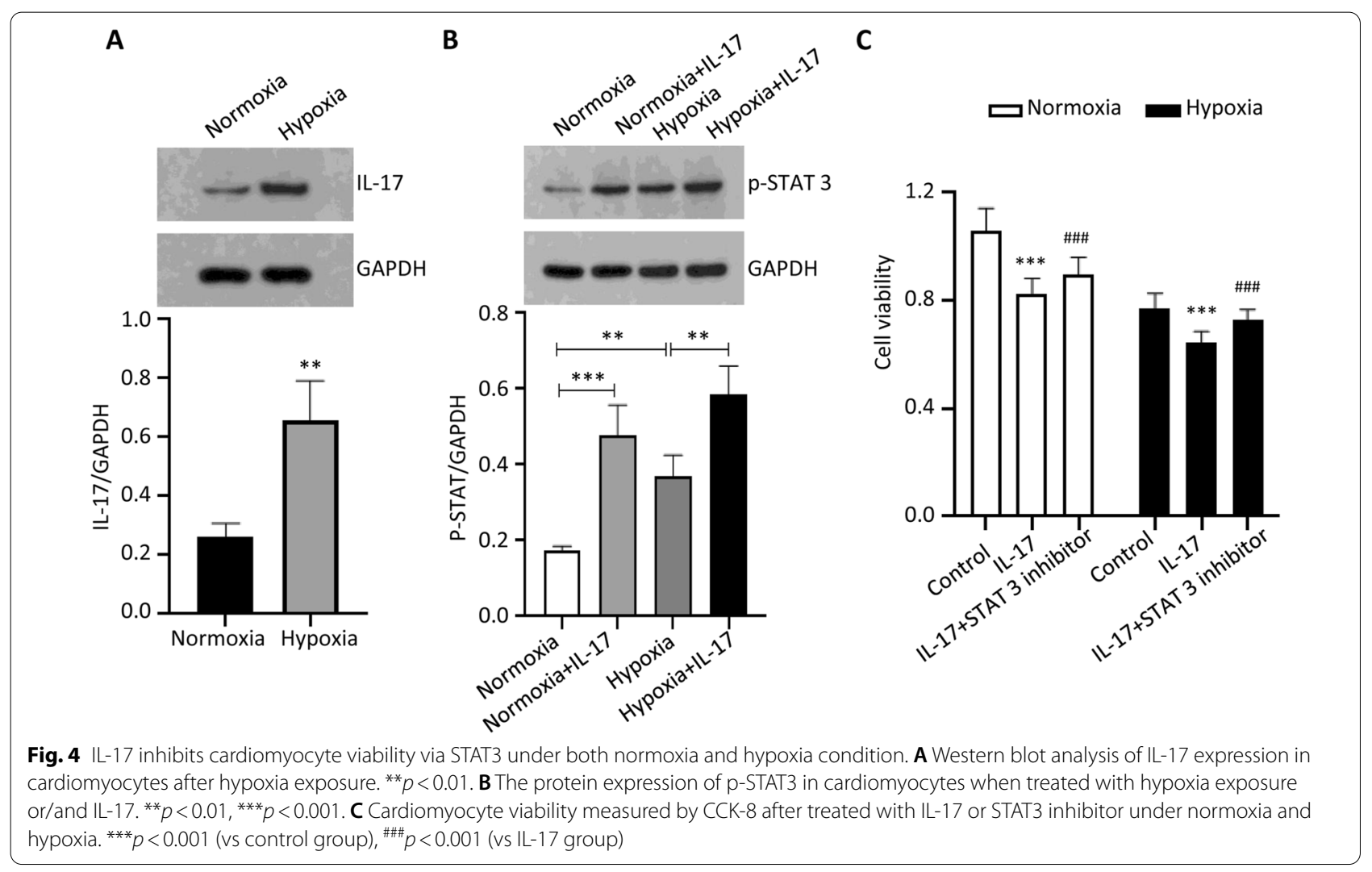


A

a

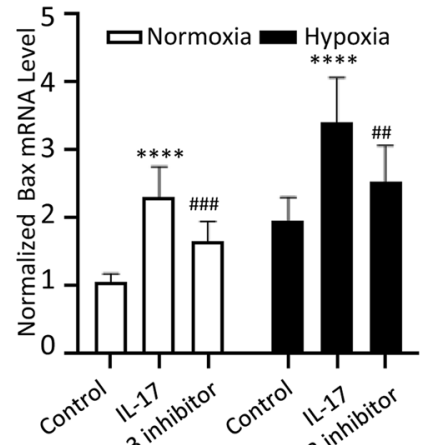

b
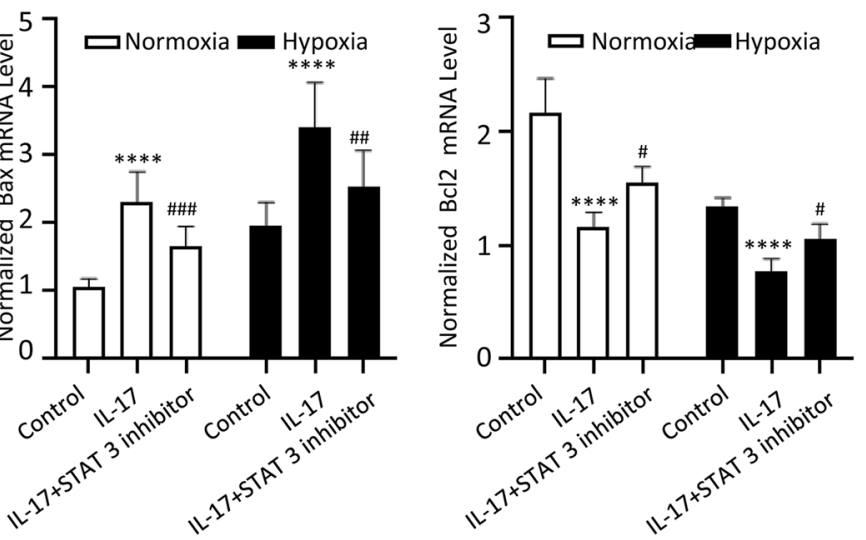

C

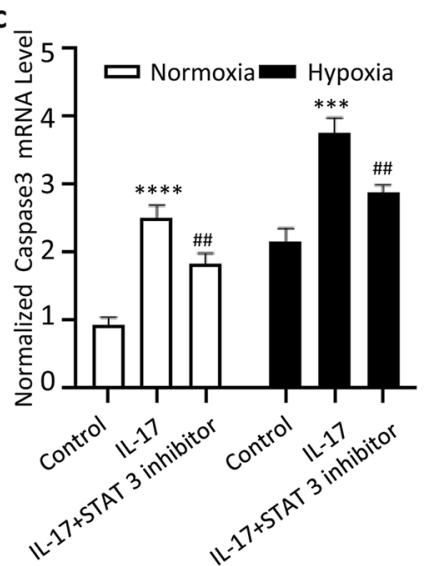

B

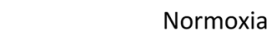

Hypoxia

b

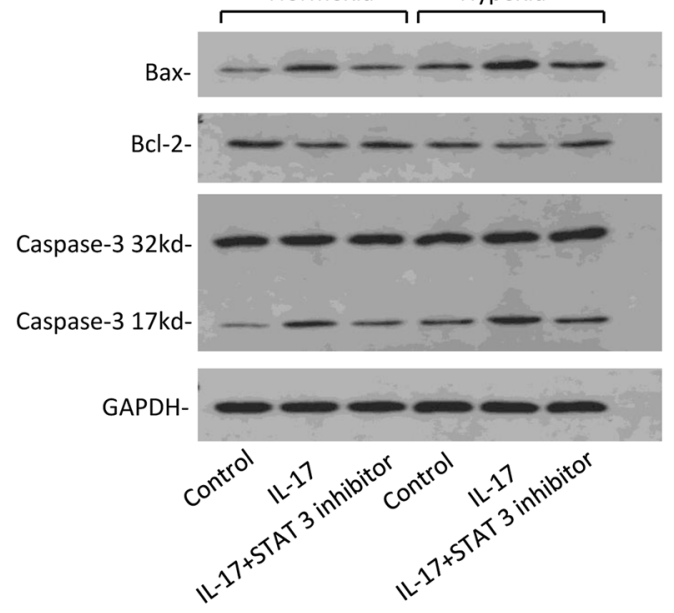

C
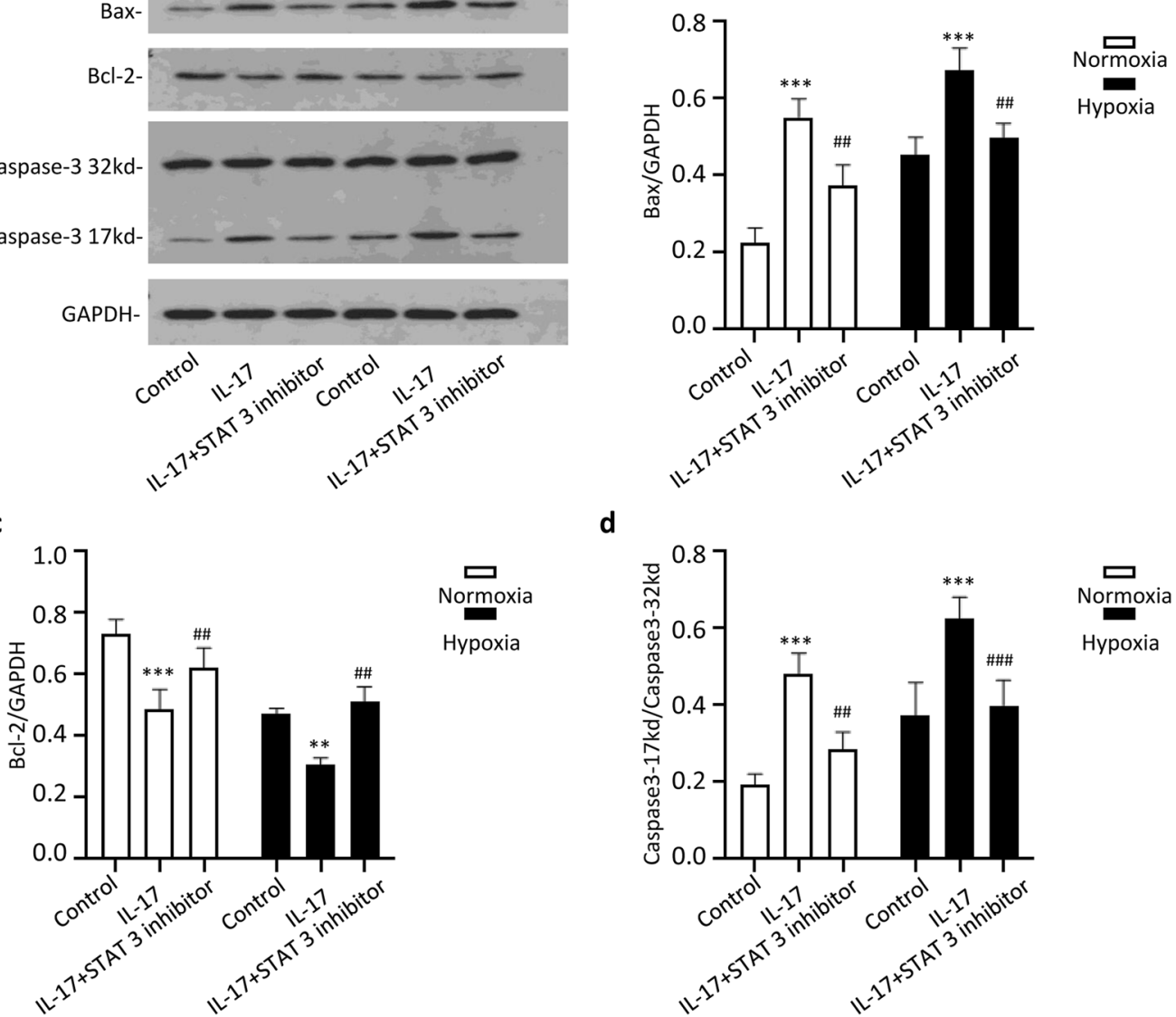

d

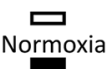

Hypoxia

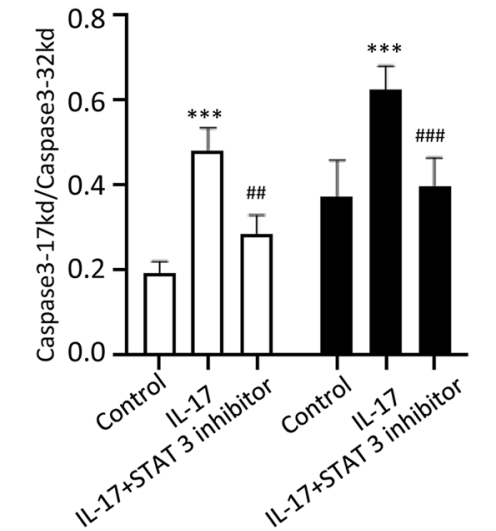

Fig. 5 IL-17 induces cardiomyocyte apoptosis via STAT3 under both normoxia and hypoxia condition. (A, a, b, c) the normalized mRNA level of Bax, Bcl-2 and caspase3 after treated with IL-17 or STAT3 inhibitor under normoxia and hypoxia, respectively. ${ }^{* *} p<0.001,{ }^{* * *} p<0.0001$ (vs control group). ${ }^{\#} p<0.05, \# p<0.01, \# \# p<0.001$ (vs IL-17 group). B the protein expression of Bax, Bcl-2 and caspase3 after treated with IL-17 or STAT3 inhibitor under normoxia and hypoxia. (a) Western blot analysis of Bax, Bcl-2 and caspase-3. (b, c, d) Statistical bar graph for expression of Bax, Bcl-2 and caspase-3 respectively. ${ }^{* *} p<0.01,{ }^{* *} p<0.001$ (vs control group). ${ }^{\# \#} p 0.01,{ }^{\# \#} p<0.001$ (vs IL-17 group) 
IL-17 is involved in RVH, suggesting a therapeutic potential target and diagnostic biomarker of IL-17 for RVH.

Several previous studies showed that IL-17 plays an important role in ventricular remodeling, but almost all of them focused on the left ventricle. In heart failure models, the levels of circulating $\mathrm{CD} 4^{+} \mathrm{T}$ cells and $\mathrm{CD}^{+} \mathrm{T}$ cells infiltration in the LV were significantly increased, and the expression of IL-17 was upregulated. Levels of fibrosis and collagen deposition were increased after IL-17 treatment [9]. In post- myocardial infarction mouse remodeling, IL-17 significantly aggravated both early- and late-phase left ventricular remodeling, but IL-17 deficiency had the opposite effect [11]. IL-17 did not participate in the early post-MI inflammatory process, but it had a specific role in the late remodeling stages by enhancing neutrophil and macrophage infiltration [19]. Additionally, the blockade of the IL-17 pathway was reported to alleviate late post-AMI remodeling [20]. In a myocarditis mouse model, IL-17 was essential for the development of the heart muscle enlargement, the heart ventricles dilatation, and the remodeling of LV during the process of transition from myocarditis to dilated cardiomyopathy $[21,22]$. An in vitro study, showed that IL-17 directly induced apoptosis of cardiomyocytes [23]. In another investigation, IL-17 induced chemokine production by cardiac fibroblasts, resulting in neutrophil and macrophage infiltration in the heart, which critically involved in the pathogenesis of inflammatory dilated cardiomyopathy [24]. In cultured cardiac fibroblasts, IL-17 promoted the proliferation of fibroblasts, and upregulated the expression of ADAMTS-1, MMP-2, and collagen subtypes I and III, leading to fibrosis and collagen deposition [25].

So far, very few studies have investigated the role of IL-17 in right heart diseases. In one of them, IL-17 was upregulated in arrhythmogenic right ventricular cardiomyopathy which was characterized by fibrofatty remodeling [26]; however, but it did not examine its role in right ventricular cardiomyopathy and the possible mechanisms. These previous results are consistent with our findings, we confirmed that the expression of IL-17 in the RV tissue during the chronic hypoxia exposure was upregulated in a time-dependent manner. Furthermore, we also found that IL-17 induced RVH under normoxia and aggravated hypoxia-induced right ventricular hypertrophy.

Cardiomyocyte hypertrophy and cardiomyocyte apoptosis are two different cellular events in ventricular hypertrophy, and many factors can cause both cardiomyocyte hypertrophy and apoptosis, and hypertrophic cardiomyocyte can turned into apoptotic cell [27, 28]. Cardiomyocyte apoptosis is a well-known key cellular event during ventricular hypertrophy [2]. While cardiomyocytes apoptosis is rare in the normal heart, apoptotic rates increase significantly in human heart failure $[29,30]$. Apoptosis rates in animal models were established to vary widely, but the potential of hypoxia to induce cardiomyocyte apoptosis is known [31]. Cardiomyocyte loss and subsequent reparative fibrotic healing lead to myocardial dysfunction and remodeling [32]. Nevertheless, previous studies showed that cardiomyocyte apoptosis can be manipulated and even reversed [33, 34]. Therefore, it is important to elucidate the mechanisms of cardiomyocyte apoptosis, which have not yet been fully clarified. In the present investigation both IL-17 and hypoxia caused cardiomyocyte apoptosis. Apoptosis-related proteins, such as $\mathrm{Bcl}-2$, Bax and caspase-3, are known to be critically involved in apoptosis. Our present results indicated that IL-17 markedly downregulated Bcl-2, upregulated Bax and caspase-3, and reduced the survival rate of cardiomyocytes. Meanwhile, the treatment with STAT-3 inhibitor reversed the aforementioned effects. These indicated that IL-17 may exert its pro-apoptotic effects through STAT-3 expression regulation.

IL-17 (usually known as IL-17A), is an important proinflammatory cytokine mainly produced by Th17 cells that binds to a heteromeric receptor, composed of IL-17RA and IL-17RC subunits, which activates the canonical NF-kB, MEK-ERK1/2, PI3K-Akt, JNK, and p38 MAPK pathways [35]. The data obtained in this study showed that the expression of p-STAT3 in IL-17-deficient mice RV tissue was downregulated, indicating that IL-17 may induce right ventricular hypertrophy through activating STAT3. Further, our present results revealed that the applied STAT-3 inhibitor reversed the pro-apoptotic effect of IL-17; hence, IL-17/STAT-3 pathway plays an important role in the cardiomyocyte apoptosis.

There are several limitations in this study. First, here, we did not compare the difference between RV and LV. Second, RV hypertrophy biomarkers, myocardial inflammation, myocardial fibrosis, right ventricular structure and function features was not assessed in this study. Third, cardiomyocyte-specific IL-17 deficiency mice, other pulmonary hypertension animal models and right ventricular failure model were not used to investigate the role of IL-17 in RVH. Last, the deep pathogenesis mechanisms need to be further investigated. Certainly, all of these are our future research directions. In our future investigations, we will isolate and culture RV and LV primary cardiomyocytes to establish the pathogenetic mechanisms of IL-17 in RVH using different animal models. 


\section{Conclusions}

In conclusion, this work provides preliminary evidence that IL-17 is a mediator in the pathogenesis RVH, which might be considered as a potential novel anti-inflammation therapeutic strategy or diagnostic biomarker for RVH.

\section{Supplementary Information}

The online version contains supplementary material available at https://doi. org/10.1186/s12872-021-02069-4.

Additional file 1. Full-length blots of WB results and flowcharts of in vivo experiments.

\section{Acknowledgements}

We thank professor Hua-ping Dai from China-Japan Friendship Hospital (Beijing, China) for providing IL-17 knockout mice.

\section{Authors' contributions}

LW and CZ designed research; HJ and WZ did the experiments and analyzed data; $\mathrm{JH}$ and LW wrote the paper. All authors reviewed the manuscript. All authors read and approved the final manuscript.

\section{Funding}

This paper was supported by National Natural Science Foundation of China (81860014), and Fundamental Research Funds for the Central Universities (xzy012020060).

\section{Availability of data and materials}

The data used to support the findings of this study are available from the corresponding author upon request.

\section{Declarations}

\section{Ethics approval and consent to participate}

The experimental animals were approved by the Institutional Animal Care and Use Committee of Xi'an jiaotong University. Mice used in this study are anesthetized in accordance with ARRIVE guidelines. This study did not involve human participants.

\section{Consent for publication}

Not required; this manuscript does not include any data relating to an individual person.

\section{Conflict of interest}

No conflicts of interest, financial or otherwise, are declared.

\section{Author details}

${ }^{1}$ Department of Rheumatism and Immunology, First Affiliated Hospital of Xi'an Jiaotong University, Xi'an 710061, People's Republic of China. ${ }^{2}$ Department of Emergency Medicine, First Affiliated Hospital of Xi'an Jiaotong University, Xi'an 710061, People's Republic of China. ${ }^{3}$ Department of Pulmonary and Critical Care Medicine, Yanan University Affiliated Hospital, Yanan 716000, People's Republic of China. ${ }^{4}$ Department of Pulmonary and Critical Care Medicine, Second Affiliated Hospital of Xi'an Jiaotong University, No. 157 Xiwu Road, Xin Cheng District, Xi'an 710004, People's Republic of China.

Received: 7 January 2021 Accepted: 17 May 2021

Published online: 21 May 2021

\section{References}

1. Simonneau G, Montani D, Celermajer DS, Denton CP, Gatzoulis MA, Krowka M, Williams PG, Souza R. Haemodynamic definitions and updated clinical classification of pulmonary hypertension. Eur Respir J. 2019;53(1).

2. Bogaard HJ, Abe K, Vonk Noordegraaf A, Voelkel NF. The right ventricle under pressure: cellular and molecular mechanisms of right-heart failure in pulmonary hypertension. Chest. 2009;135(3):794-804.

3. Rabinovitch M, Guignabert C, Humbert M, Nicolls MR. Inflammation and immunity in the pathogenesis of pulmonary arterial hypertension. Circ Res. 2014;115(1):165-75.

4. Mann DL. Inflammatory mediators and the failing heart: past, present, and the foreseeable future. Circ Res. 2002;91(11):988-98.

5. Perticone M, Zito R, Miceli S, Pinto A, Suraci E, Greco M, Gigliotti S, Hribal ML, Corrao S, Sesti G, et al. Immunity, inflammation and heart failure: their role on cardiac function and iron status. Front Immunol. 2019;10:2315.

6. Shirazi LF, Bissett J, Romeo F, Mehta JL. Role of inflammation in heart failure. Curr Atheroscler Rep. 2017;19(6):27.

7. Chiurchiu V, Leuti A, Saracini S, Fontana D, Finamore P, Giua R, Padovini L, Incalzi RA, Maccarrone M. Resolution of inflammation is altered in chronic heart failure and entails a dysfunctional responsiveness of T lymphocytes. FASEB J. 2019;33(1):909-16.

8. Timmers L, Sluijter JP, van Keulen JK, Hoefer IE, Nederhoff MG, Goumans MJ, Doevendans PA, van Echteld CJ, Joles JA, Quax PH, et al. Toll-like receptor 4 mediates maladaptive left ventricular remodeling and impairs cardiac function after myocardial infarction. Circ Res. 2008;102(2):257-64.

9. Chang SL, Hsiao YW, Tsai YN, Lin SF, Liu SH, Lin YJ, Lo LW, Chung FP, Chao TF, Hu YF, et al. Interleukin-17 enhances cardiac ventricular remodeling via activating MAPK pathway in ischemic heart failure. J Mol Cell Cardiol. 2018;122:69-79.

10. Hofmann U, Beyersdorf N, Weirather J, Podolskaya A, Bauersachs J, Ertl G, Kerkau T, Frantz S. Activation of CD4+ T lymphocytes improves wound healing and survival after experimental myocardial infarction in mice. Circulation. 2012;125(13):1652-63.

11. Zhou SF, Yuan J, Liao MY, Xia N, Tang TT, Li JJ, Jiao J, Dong WY, Nie SF, Zhu ZF, et al. IL-17A promotes ventricular remodeling after myocardial infarction. J Mol Med (Berl). 2014;92(10):1105-16.

12. Peng Y, Zhou B, Wang YY, Shi S, Zhang K, Zhang L, Rao L. Analysis of IL-17 gene polymorphisms in Chinese patients with dilated cardiomyopathy. Hum Immunol. 2013;74(5):635-9.

13. Wang L, Liu J, Wang W, Qi X, Wang Y, Tian B, Dai H, Wang J, Ning W, Yang T, et al. Targeting IL-17 attenuates hypoxia-induced pulmonary hypertension through downregulation of beta-catenin. Thorax. 2019;74(6):564-78.

14. Liu J, Wang W, Wang L, Qi XM, Sha YH, Yang T. 3-Bromopyruvate alleviates the development of monocrotaline-induced rat pulmonary arterial hypertension by decreasing aerobic glycolysis, inducing apoptosis, and suppressing inflammation. Chin Med J (Engl). 2020;133(1):49-60.

15. Wang W, Liu J, Ma A, Miao R, Jin Y, Zhang H, Xu K, Wang C, Wang J. mTORC1 is involved in hypoxia-induced pulmonary hypertension through the activation of Notch3. J Cell Physiol. 2014;229(12):2117-25.

16. Liu J, Wang W, Wang L, Chen S, Tian B, Huang K, Corrigan CJ, Ying S, Wang W, Wang C. IL-33 initiates vascular remodelling in hypoxic pulmonary hypertension by up-regulating HIF-1alpha and VEGF expression in vascular endothelial cells. EBioMedicine. 2018;33:196-210.

17. Faber JE, Szymeczek CL, Cotecchia S, Thomas SA, Tanoue A, Tsujimoto G, Zhang H. Alpha1-adrenoceptor-dependent vascular hypertrophy and remodeling in murine hypoxic pulmonary hypertension. Am J Physiol Heart Circ Physiol. 2007;292(5):H2316-2323.

18. Song XL, Zhang FF, Wang WJ, Li XN, Dang Y, Li YX, Yang Q, Shi MJ, Qi $X Y$. LnCRNA A2M-AS1 lessens the injury of cardiomyocytes caused by hypoxia and reoxygenation via regulating IL1R2. Genes Genom. 2020;42(12):1431-41.

19. Yan $X$, Shichita T, Katsumata $Y$, Matsuhashi T, Ito H, Ito K, Anzai A, Endo J, Tamura Y, Kimura K, et al. Deleterious effect of the IL-23/IL-17A axis and gammadelta $T$ cells on left ventricular remodeling after myocardial infarction. J Am Heart Assoc. 2012;1(5):e004408.

20. Yan X, Anzai A, Katsumata Y, Matsuhashi T, Ito K, Endo J, Yamamoto T, Takeshima A, Shinmura K, Shen W, et al. Temporal dynamics of cardiac immune cell accumulation following acute myocardial infarction. J Mol Cell Cardiol. 2013;62:24-35.

21. Nind V, Maier R, Ratering D, De Giuli R, Zust R, Thiel V, Scandella E, Di Padova F, Kopf M, Rudin M, et al. Cooperation of Th1 and Th17 cells 
determines transition from autoimmune myocarditis to dilated cardiomyopathy. Eur J Immunol. 2012;42(9):2311-21.

22. Baldeviano GC, Barin JG, Talor MV, Srinivasan S, Bedja D, Zheng D, Gabrielson K, Iwakura Y, Rose NR, Cihakova D. Interleukin-17A is dispensable for myocarditis but essential for the progression to dilated cardiomyopathy. Circ Res. 2010;106(10):1646-55.

23. Su SA, Yang D, Zhu W, Cai Z, Zhang N, Zhao L, Wang JA, Xiang M. Interleukin-17A mediates cardiomyocyte apoptosis through Stat3-iNOS pathway. Biochim Biophys Acta. 2016;1863(11):2784-94.

24. Wu L, Ong S, Talor MV, Barin JG, Baldeviano GC, Kass DA, Bedja D, Zhang H, Sheikh A, Margolick JB, et al. Cardiac fibroblasts mediate IL-17A-driven inflammatory dilated cardiomyopathy. J Exp Med. 2014;211(7):1449-64.

25. Xie Y, Li M, Wang X, Zhang X, Peng T, Yang Y, Zou Y, Ge J, Chen H, Chen $R$. In vivo delivery of adenoviral vector containing interleukin-17 receptor a reduces cardiac remodeling and improves myocardial function in viral myocarditis leading to dilated cardiomyopathy. PLoS ONE. 2013;8(8):e72158.

26. Asimaki A, Tandri H, Duffy ER, Winterfield JR, Mackey-Bojack S, Picken MM, Cooper LT, Wilber DJ, Marcus FI, Basso C, et al. Altered desmosomal proteins in granulomatous myocarditis and potential pathogenic links to arrhythmogenic right ventricular cardiomyopathy. Circ Arrhythm Electrophysiol. 2011;4(5):743-52.

27. Higuchi Y, Otsu K, Nishida K, Hirotani S, Nakayama H, Yamaguchi O, Matsumura Y, Ueno H, Tada M, Hori M. Involvement of reactive oxygen species-mediated NF-kappa B activation in TNF-alpha-induced cardiomyocyte hypertrophy. J Mol Cell Cardiol. 2002;34(2):233-40.

28. Barlucchi L, Leri A, Dostal DE, Fiordaliso F, Tada H, Hintze TH, Kajstura J, Nadal-Ginard B, Anversa P. Canine ventricular myocytes possess a renin-angiotensin system that is upregulated with heart failure. Circ Res. 2001;88(3):298-304.
29. Olivetti G, Abbi R, Quaini F, Kajstura J, Cheng W, Nitahara JA, Quaini E, Di Loreto C, Beltrami CA, Krajewski S, et al. Apoptosis in the failing human heart. N Engl J Med. 1997;336(16):1131-41.

30. Kang PM, Izumo S. Apoptosis and heart failure: a critical review of the literature. Circ Res. 2000;86(11):1107-13.

31. Wei L, Zhou Q, Tian H, Su Y, Fu GH, Sun T. Integrin beta3 promotes cardiomyocyte proliferation and attenuates hypoxia-induced apoptosis via regulating the PTEN/Akt/mTOR and ERK1/2 pathways. Int J Biol Sci. 2020;16(4):644-54.

32. Schirone L, Forte M, Palmerio S, Yee D, Nocella C, Angelini F, Pagano F, Schiavon S, Bordin A, Carrizzo A, et al. A review of the molecular mechanisms underlying the development and progression of cardiac remodeling. Oxid Med Cell Longev. 2017;2017:3920195.

33. Long B, Gan TY, Zhang RC, Zhang YH. miR-23a regulates cardiomyocyte apoptosis by targeting manganese superoxide dismutase. Mol Cells. 2017:40(8):542-9.

34. Hohensinner PJ, Takacs N, Kaun C, Thaler B, Krychtiuk KA, Pfaffenberger S, Aliabadi A, Zuckermann A, Huber K, Wojta J. Urokinase plasminogen activator protects cardiac myocytes from oxidative damage and apoptosis via hOGG1 induction. Apoptosis. 2017;22(8):1048-55.

35. Gaffen SL. Structure and signalling in the IL-17 receptor family. Nat Rev Immunol. 2009;9(8):556-67.

\section{Publisher's Note}

Springer Nature remains neutral with regard to jurisdictional claims in published maps and institutional affiliations.
Ready to submit your research? Choose BMC and benefit from:

- fast, convenient online submission

- thorough peer review by experienced researchers in your field

- rapid publication on acceptance

- support for research data, including large and complex data types

- gold Open Access which fosters wider collaboration and increased citations

- maximum visibility for your research: over 100M website views per year

At BMC, research is always in progress.

Learn more biomedcentral.com/submissions 